\title{
Parathyroid Cyst containing an Adenoma: A Rare Cervical Lesion causing Hyperparathyroidism
}

\author{
${ }^{1}$ Angeliki Chorti, ${ }^{2}$ loannis Pliakos, ${ }^{3}$ Stavros Panidis, ${ }^{4}$ Triantafyllia Koletsa, ${ }^{5}$ Tzikos Georgios, ${ }^{6}$ Mouslech Zadala \\ ${ }^{7}$ Christina Manani, ${ }^{8}$ Antonios Michalopoulos, ${ }^{9}$ Theodossis S Papavramidis
}

\begin{abstract}
Aim and introduction: Parathyroid cysts (PCs) are lesions arising from the parathyroid glands and are located from the angle of the mandible to the mediastinum, representing 1 to $5 \%$ of neck masses. They have a female predominance and occur usually in the fourth and fifth decade of life. They are subdivided into two categories according to their hormonal activity: Functioning and nonfunctioning ones. Except for parathyroid adenoma, hyperplasia, and carcinoma, functioning cysts are an additional rare cause of primary hyperparathyroidism $(\mathrm{PH})$ and should be taken into consideration in the investigation of cystic neck masses.
\end{abstract}

Case report: We report a case of 38-year-old man, who was admitted to our hospital for the investigation of an asymptomatic hypercalcemia identified incidentally. In his laboratory tests, ionized calcium and parathormone (PTH) were elevated, while phosphorus was decreased. A $6.5 \times 3.5 \mathrm{~cm}$ cystic structure was revealed in ultrasound examination, scintigraphy, and magnetic resonance imaging (MRI) scan, but was not indicative of PC, and surgical exploration was mandatory. The cyst was completely excised and the pathological examination revealed a PC that coexisted with an adenoma. Postoperative PTH, calcium, and phosphorus values were within normal ranges and the patient was discharged the first postoperative day.

Conclusion and clinical significance: Parathyroid cysts should be treated with gentle manipulations during surgical procedure in order that it is not ruptured and the recurrence is avoided.

Keywords: Hyperparathyroidism, Parathyroid adenoma, Parathyroid cyst, Parathyroid glands.

How to cite this article: Chorti A, Pliakos I, Panidis S, Koletsa T, Georgios K, Zadala M, Manani C, Michalopoulos A, Papavramidis TS. Parathyroid Cyst containing an Adenoma:

\footnotetext{
${ }^{1-3,5,8,9}$ Propedeutic, ${ }^{4}$ Pathologist, ${ }^{6,7}$ Endocrinologist

1-3,5,8,9 Department of Surgery, School of Medicine, Aristotle University of Thessaloniki, AHEPA University General Hospital of Thessaloniki, Thessaloniki, Greece

${ }^{4}$ Department of Pathology, Aristotle University of Thessaloniki Thessaloniki, Greece

${ }^{6}$ Department of Internal Medicine, School of Medicine, Aristotle University of Thessaloniki, AHEPA University General Hospital of Thessaloniki, Thessaloniki, Greece

${ }^{7}$ Department of Endocrinology, School of Medicine, Aristotle University of Thessaloniki, Ippokrateio University Hospital of Thessaloniki, Thessaloniki, Greece

Corresponding Author: Theodossis S Papavramidis Propedeutic, Department of Surgery, School of Medicine Aristotle University of Thessaloniki, AHEPA University General Hospital of Thessaloniki, Thessaloniki, Greece, Phone: +306944536972, e-mail: papavramidis@hotmail.com
}

A Rare Cervical Lesion causing Hyperparathyroidism. World J Endoc Surg 2018;10(1):5-7.

Source of support: Nil

Conflict of interest: None

\section{INTRODUCTION}

Primary hyperparathyroidism is a condition caused by the excessive secretion of PTH due to one or more parathyroid glands' pathology. The most common causes of $\mathrm{PH}$ are adenoma, hyperplasia, or carcinoma of parathyroid glands.

Parathyroid cysts are rare lesions that can be an additional cause of $\mathrm{PH}$. They were first described by Sandstörm in 1880 . They represent 1 to $5 \%$ of neck masses and $<0.5 \%$ of parathyroid glands' pathologies. In this report, we present and discuss a case of a large functioning PC, containing an adenoma, as a rare cause of $\mathrm{PH}$.

\section{CASE REPORT}

A 38-year-old man was admitted to our department for investigation of asymptomatic hypercalcemia identified incidentally. His past medical history and family history was negative. He took no medication. Physical examination showed no significant findings. Laboratory data had elevated serum ionized Ca $12.65 \mathrm{mg} / \mathrm{dL}$ (normal range: $8.2-10.6 \mathrm{mg} / \mathrm{dL}$ ), decreased phosphate $2.4 \mathrm{mg} / \mathrm{dL}$ (normal range: $2.7-4.5 \mathrm{mg} / \mathrm{dL}$ ), and elevated serum PTH $20.6 \mathrm{pmol} / \mathrm{L}$ (normal range: 1.58-6.03 pmol/L). Thyroid ultrasound examination revealed a low-density, avascular lesion with well-defined margins, located nearby the left inferior parathyroid gland, containing both fluid and solid parts and measuring $5.71 \times 3.73 \mathrm{~cm}$. These findings were not the typical ones for parathyroid adenoma or hyperplasia that were initially included in the differential diagnosis of hypercalcemia, so we performed a parathyroid scintigraphy with Tc99m sestamibi which has shown no isonitrile uptake by this lesion, suggesting that there is no thyroid or parathyroid tissue in it. Magnetic resonance imaging scan was mandatory in order to distinguish this neck mass. It revealed a benign cystic lesion of $3.5 \times 6.5 \mathrm{~cm}$ in size that located in the left lower lobe of the thyroid gland, extended to the superior mediastinum and displaced the adjusted structures. The surgical excision of this lesion was decided. During the operation, the lesion was identified under the left lower thyroid lobe and 

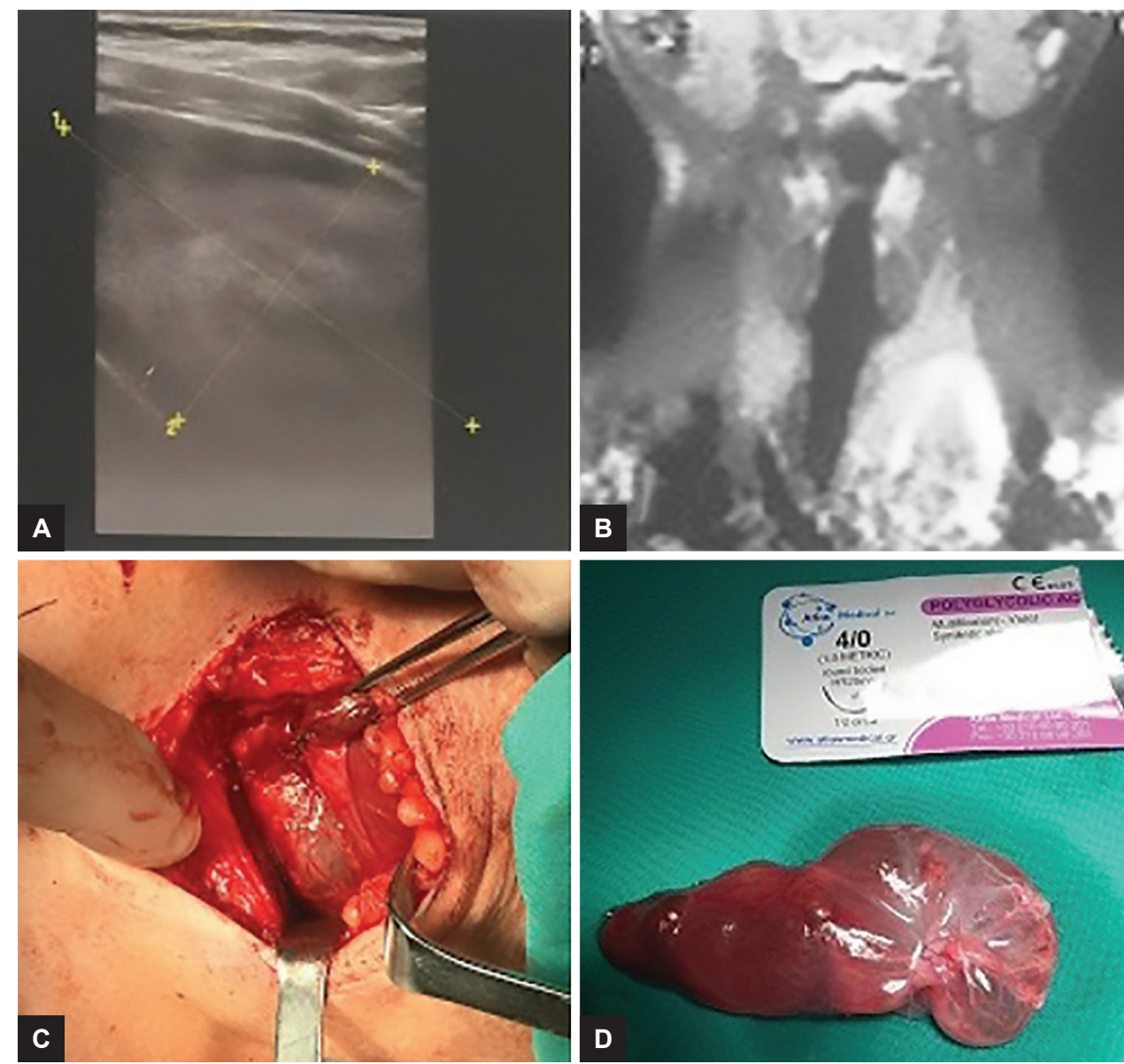

Figs 1A to D: (A) Ultrasound image of PC; (B) MRI showing a huge cystic lesion under the left thyroid lobe; (C) PC as identified during the operation; and (D) excised PC

was excised with gentle surgical manipulations in order that the rupture is avoided. Images of the diagnostic and therapeutic algorithm followed are shown in Figure 1. The patient's postoperative course was uneventful, while postoperative serum PTH and ionized Ca were within normal range $(0.7 \mathrm{pmol} / \mathrm{L}$ and $10.5 \mathrm{mg} / \mathrm{dL}$ respectively). The patient was discharged the first postoperative day. Pathology revealed hyperplastic parathyroid tissue with several sites of normal tissue surrounded by connective tissue, while the inner surface of the cystic wall was also structured by hyperplastic tissue. These findings were indicative of PC that contained an adenoma.

\section{DISCUSSION}

Parathyroid cysts are rare lesions that arise in the neck and the mediastinum. According to several studies, PCs have a female predilection, as the ratio between male and female patients is 1:2.5, while the peak incidence of PCs is between 30 and 50 years old. ${ }^{1,2}$ Parathyroid cysts are subdivided into two categories: Functioning and nonfunctioning, according to their hormonal activity.

The pathogenesis of PCs remains controversial. Several theories about the etiology of these cysts have been reported: (1) remnants of pharyngobranchial ducts, (2) persistence of Kürsteiner canals, (3) remnants of third and fourth branchial cleft, (4) retention of secretions of PTH in cysts, (5) coalescence of clefts, (6) cystic degeneration or hemorrhage in an adenoma or hyperplastic gland, which is mainly referred to functioning cysts. ${ }^{3}$

Parathyroid cysts can be found from the angle of the mandible to the mediastinum. A higher incidence of PCs as neck masses is reported in the international literature, while in the mediastinum they are most commonly found in the superior part followed by the anterior. Furthermore, there are references in the literature of intrathyroidal and intrathymic cysts as additional locations of PCs. ${ }^{3}$

Regarding symptoms, PCs can be found as an asymptomatic palpable neck mass, can be an incidental finding, or can cause compressive symptoms. Cases of vocal cord paralysis as a result of the cyst compression have been reported in the literature. As far as the functioning of PCs is concerned, they cause $\mathrm{PH}^{2}$

The diagnosis of PCs is based on imaging techniques and especially on ultrasound and computed tomography (CT) and MRI. In order to elucidate the topography, CT and MRI scan are reserved for larger cysts. Ultrasound examination is an easy method to reveal the cystic nature 
of the mass and its dimensions, while it is usually combined with fine-needle aspiration (FNA). ${ }^{2}$

A wide variety of neck and mediastinal masses are included in the differential diagnosis of PCs. Thyroid nodules are the main category of neck masses from which PCs should be differentiated. Furthermore, thyroid cyst, thymic cyst, thyroid adenoma, and parathyroid carcinoma should be taken into consideration in case of investigation of neck mass. ${ }^{4}$ The cystic fluid aspiration is the appropriate tool to distinguish a thyroid from a PC. Thymic cyst, thyroglossal duct cyst, and branchial cleft cyst are further cystic structures included in the differential diagnosis of PCs, but they are most commonly found in children as congenital conditions. ${ }^{4,5}$ In the mediastinum, PCs should be differentiated from vascular lesions, teratomas, adenopathy, lipomas, neuromas, bronchogenic cysts, thymomas, lymphangiomas, cystic hygromas, masses of esophagus, trachea, nerves, lymphatics, thyroid, and parathyroid glands. ${ }^{6}$

The suggested initial treatment of PCs is FNA, especially in case of nonfunctioning cysts. If the cysts recur, then surgical excision is the definitive treatment. In the literature, there are several reports of the usage of ethanol or tetracycline injection, especially in cases of nonfunctioning cysts. ${ }^{7}$ It is recommended that the nonfunctioning cysts should be treated with FNA, whereas the functioning cysts should be surgically excised. ${ }^{2}$ The main indications for surgery are: (1) hyperparathyroidism, (2) uncertain diagnosis, (3) possibility for malignancy, (4) symptomatology, (5) cases of recurrence. ${ }^{8}$

Regarding pathology, PCs are lined by cuboidal or columnar epithelium and are thin-walled white cysts, while the dominant characteristic is the parathyroid tissue found in the epithelium. ${ }^{9}$ In case of functioning PCs, the epithelium is missing and they look like pseudocysts. The cytological analysis of the fluid reveals clusters of epithelial cells and fibroblasts atypically proliferated that can be mistaken for malignant cells. The fluid is clear-watery and in many cases of adenoma, it can be hemorrhagic. ${ }^{1}$

The prognosis of PCs seems to be excellent. There are no reports of malignancy of PCs or deaths due to PCs in the literature.

\section{REFERENCES}

1. Grunberg WI, Domingo OH. Parathyroid cyst. Am Surg 2011 Nov;77(11):E256-E257.

2. Pontikides N, Karras S, Kaprara A, Cheva A, Doumas A, Botsios D, Moschidis A, Efthimiou E, Wass J, Krassas G. Diagnostic and therapeutic review of cystic parathyroid lesions. Hormones (Athens) 2012 Oct-Dec;11(4):410-418.

3. Agrawal D, Lahiri TK, Agrawal A, Singh MK. Uncommon parathyroid mediastinal cyst compressing the trachea. Indian J Chest Dis Allied Sci 2006 Oct-Dec;48(4):279-281.

4. Arduc A, Tutuncu YA, Dogan BA, Ileri ABA, Tuna MM, Ozcan HN, Isik S, Berker D, Guler S. Parathyroid cysts. Am Surg 2015 Apr;81(4):E163-E165.

5. Kato H, Kanematsu M, Kiryu T, Iwata H, Shirahashi K, Matsumoto S, Hirose Y, Matsutomo H, Sasaoka I. Nonfunctional mediastinal parathyroid cyst: imaging findings in two cases. Clin Imaging 2008 Jul-Aug;32(4):310-313.

6. Hoskuldsdottir A, Kristvinsson H, Gudjonsson H, Geirsson A, Gudbjartsson T. Mediastinal parathyroid cyst-a case report. Laeknabladid 2014 Sep;100(9):453-455.

7. Karras SN, Koutelidakis I, Anagnostis P, Mintziori G, Pontikides N, Goulis DG. A rare case of a parathyroid adenoma inside a parathyroid cyst. Arq Bras Endocrinol Metabol 2014 Oct;58(7):776-778.

8. Kassaras GA, Nissotakis C, Spiridakis J, Toloumis G, Dritsakos K, Sakorafas GH. Parathyroid gland cyst. Am Surg 2010 Apr;76(4):11-12.

9. Ghervan C, Goel P. Parathyroid cyst, a rare cause of cystic cervical lesion. Case report. Med Ultrason 2011 Jun;13(2):157-160. 